\title{
Supplementary Material for "A Multi-Resolution Model for Non-Gaussian Random Fields on a Sphere with Application to Ionospheric Electrostatic Potentials"
}

\author{
Minjie Fan ${ }^{* 1}$, Debashis Paul ${ }^{1}$, Thomas C. M. Lee ${ }^{1}$, and Tomoko Matsuo ${ }^{2}$ \\ ${ }^{1}$ Department of Statistics, University of California, Davis \\ ${ }^{2}$ Department of Aerospace Engineering Sciences, University of Colorado, Boulder
}

\section{S.1 Numerical Experiment Details}

\section{S.1.1 Specification of $b(\cdot)$}

To make the paper self-contained, this subsection gives the details of the specification of the function $b(\cdot)$ in the construction of spherical needlets. We follow the second recipe in Marinucci and Peccati (2011, Chapter 10.2.2) so that $b(\cdot) \in C^{\infty}$ and satisfies the three conditions (i), (ii) and (iii) for the Littlewood-Paley decomposition. This specification can be summarized as follows:

1. Construct

$$
\phi_{1}(t)=\left\{\begin{array}{ll}
\exp \left(-\frac{1}{1-t^{2}}\right), & -1 \leq t \leq 1 \\
0, & \text { otherwise }
\end{array} .\right.
$$

2. Normalizing $\phi_{1}$, we have

$$
\phi_{2}(u)=\frac{\int_{-1}^{u} \phi_{1}(t) d t}{\int_{-1}^{1} \phi_{1}(t) d t} .
$$

3. Applying a change of variables to $\phi_{2}$, we construct

$$
\phi_{3}(t)= \begin{cases}1, & 0 \leq t \leq \frac{1}{B} \\ \phi_{2}\left(1-\frac{2 B}{B-1}\left(t-\frac{1}{B}\right)\right), & \frac{1}{B}<t \leq 1 . \\ 0, & t>1\end{cases}
$$

\footnotetext{
${ }^{*}$ Corresponding author. Email: mjfan@ucdavis.edu.
} 
4. Finally, we specify

$$
b(x)=\sqrt{\phi_{3}\left(\frac{x}{B}\right)-\phi_{3}(x)}, \quad-\infty<x<\infty .
$$

\section{S.1.2 Specification of $f(j)$}

In this subsection, we justify the specification of $f(j)$ through computing the variance of the needlet coefficients for a zero-mean and two-weakly isotropic random field on the unit sphere, denoted by $\left\{X(\mathbf{s}), \mathbf{s} \in \mathbb{S}^{2}\right\}$. According to Marinucci and Peccati (2011, Theorem 5.13), the random field has the Karhunen-Loève expansion

$$
X(\mathbf{s})=\sum_{l=0}^{\infty} \sum_{m=-l}^{l} a_{l m} Y_{l m}(\mathbf{s})
$$

where

$$
a_{l m}=\int_{\mathbb{S}^{2}} X(\mathbf{s}) \bar{Y}_{l m}(\mathbf{s}) d \mathbf{s}
$$

Moreover,

$$
E\left(a_{l m} \bar{a}_{l^{\prime} m^{\prime}}\right)=C_{l} \delta_{l l^{\prime}} \delta_{m m^{\prime}}
$$

where $\delta_{a b}=1$ if $a=b$, and 0 otherwise, and $\left\{C_{l}, l \geq 0\right\}$ are called the angular power spectrum of $X$. In our context, the needlets are constructed on the symmetric spherical t-designs (Womersley, 2015). Thus, the quadrature weights $\lambda_{j k} \equiv \lambda_{j}=4 \pi / p_{j}$, where the number of needlets at frequency level $j$

$$
p_{j}=\frac{1}{2}\left(2\left\lfloor B^{j+1}\right\rfloor\right)^{2}+\frac{1}{2} \cdot 2\left\lfloor B^{j+1}\right\rfloor+\mathcal{O}(1)=2 B^{2 j+2}+\mathcal{O}(1) .
$$

Recall that the needlet coefficients of $X$ are defined as

$$
\beta_{j k}=\int_{\mathbb{S}^{2}} X(\mathbf{s}) \psi_{j k}(\mathbf{s}) d \mathbf{s} .
$$

After certain algebraic manipulations, we then have

$$
\operatorname{Var}\left(\beta_{j k}\right)=\lambda_{j} \sum_{l} b^{2}\left(\frac{l}{B^{j}}\right) C_{l}\left(\frac{2 l+1}{4 \pi}\right) .
$$

It is natural to assume that $C_{l}$ decays in the order of $l^{-\alpha}$, where $\alpha>2$, i.e., there exist certain constants $c_{1}$ and $c_{2}$ such that $c_{1} l^{-\alpha} \leq C_{l} \leq c_{2} l^{-\alpha}$ (Baldi et al., 2009). Under this assumption and based on the proof of Baldi et al. (2009, Lemma 3), we have

$$
c_{1} B^{(2-\alpha) j} \leq \sum_{l} b^{2}\left(\frac{l}{B^{j}}\right) C_{l}\left(\frac{2 l+1}{4 \pi}\right) \leq c_{2} B^{(2-\alpha) j} .
$$


Thus,

$$
\operatorname{Var}\left(\beta_{j k}\right)=\frac{4 \pi}{p_{j}} \sum_{l} b^{2}\left(\frac{l}{B^{j}}\right) C_{l}\left(\frac{2 l+1}{4 \pi}\right)=\mathcal{O}\left(B^{-\alpha j}\right),
$$

and accordingly, $f(j)$ is specified as $B^{-\alpha j / 2}$.

\section{S.1.3 Computing CRPS Based on MCMC Output}

This subsection describes the computation of the continuous ranked probability score (CRPS, Gneiting and Raftery (2007)) based on the MCMC samples $\left\{\boldsymbol{\theta}^{(l)}, \mathbf{c}^{(l)}, l=1, \cdots, L\right\}$ from $[\boldsymbol{\theta}, \mathbf{c} \mid \mathbf{Z}=\mathbf{z}]$. For the prediction at some location $\mathbf{s}$, its CRPS is defined as

$$
\operatorname{CRPS}\left(F_{\mathbf{s}}, z(\mathbf{s})\right)=\int_{-\infty}^{\infty}\left(F_{\mathbf{s}}(x)-\mathbb{1}\{x \geq z(\mathbf{s})\}\right)^{2} d x
$$

where $F_{\mathbf{s}}$ is the predictive cumulative distribution function $(\mathrm{CDF})$ of $Z(\mathbf{s}) \mid \mathbf{Z}=\mathbf{z}, z(\mathbf{s})$ is the actual observed value at $\mathbf{s}$, and $\mathbb{1}\{\cdot\}$ is the indicator function. Since (1) does not have a closed form in our context, an approximation method is needed to evaluate the CRPS. We follow the suggestion of Krüger et al. (2016), which uses the mixture-of-parameters (MP) technique. Specifically, $F_{\mathbf{s}}$ can be approximated by

$$
\widehat{F}_{L}^{\mathrm{MP}}(x)=\frac{1}{L} \sum_{l=1}^{L} F_{\mathbf{s}}\left(x \mid \boldsymbol{\theta}^{(l)}, \mathbf{c}^{(l)}\right)
$$

where $F_{\mathbf{s}}\left(x \mid \boldsymbol{\theta}^{(l)}, \mathbf{c}^{(l)}\right)$ is the predictive CDF of $Z(\mathbf{s}) \mid \boldsymbol{\theta}^{(l)}, \mathbf{c}^{(l)}, \mathbf{Z}=\mathbf{z}$, which follows

$$
\mathcal{N}\left(\mu^{(l)},\left(\tau^{(l)}\right)^{2}\right)
$$

with

$$
\mu^{(l)}=g^{(l)}(\mathbf{s}) \sum_{j, k} c_{j k}^{(l)} \psi_{j k}(\mathbf{s})
$$

Plugging (2) into (1) and after certain algebraic manipulations, we obtain the following approximation to the CRPS (Grimit et al., 2006)

$\operatorname{CRPS}\left(\widehat{F}_{L}^{\mathrm{MP}}, z(\mathbf{s})\right)=\frac{1}{L} \sum_{l=1}^{L} A\left(z(\mathbf{s})-\mu^{(l)},\left(\tau^{(l)}\right)^{2}\right)-\frac{1}{2 L^{2}} \sum_{l=1}^{L} \sum_{m=1}^{L} A\left(\mu^{(l)}-\mu^{(m)},\left(\tau^{(l)}\right)^{2}+\left(\tau^{(m)}\right)^{2}\right)$,

where $A\left(\mu, \tau^{2}\right)=2 \tau \phi_{\text {norm }}(\mu / \tau)+\mu\left(2 \Phi_{\text {norm }}(\mu / \tau)-1\right)$, with $\phi_{\text {norm }}$ and $\Phi_{\text {norm }}$ denoting the probability density function and the $\mathrm{CDF}$ of the standard normal distribution, respectively. 

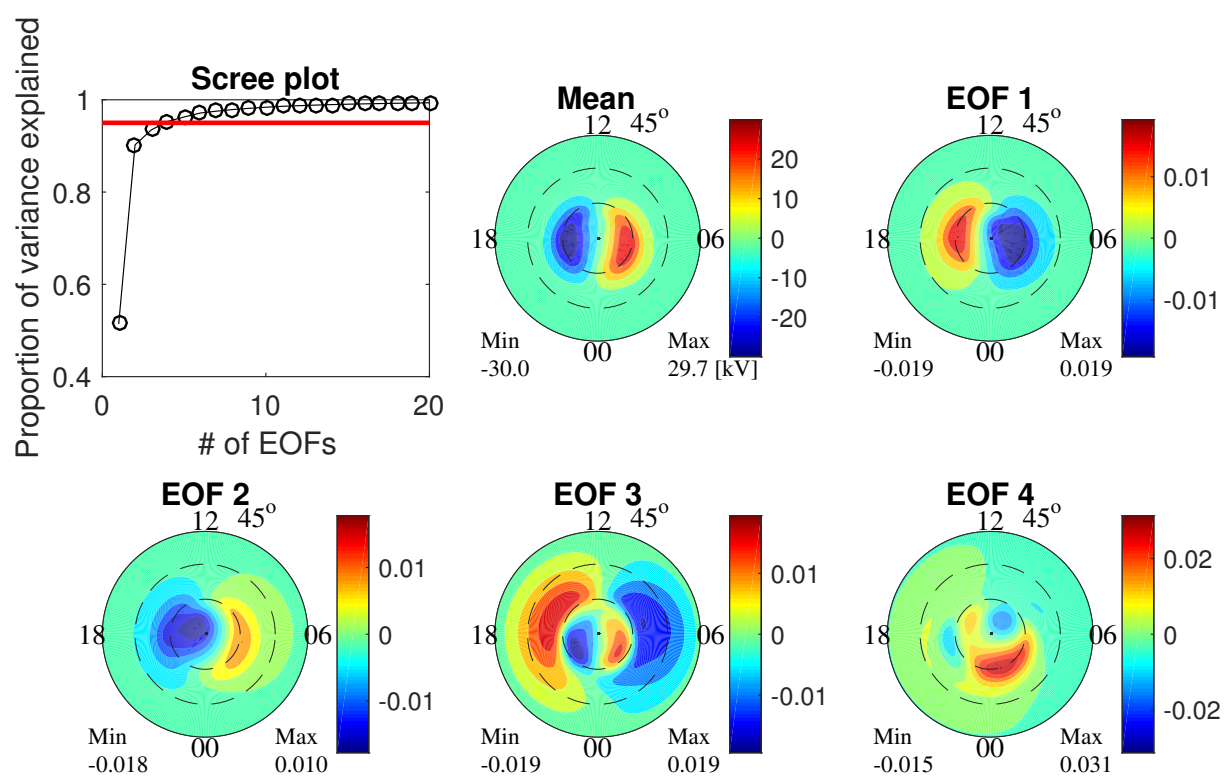

Figure 1: Results of the EOF analysis. The first four EOFs can explain approximately $95 \%$ of the total variability in the data.

\section{S.2 Application Details}

\section{S.2.1 Empirical Orthogonal Function (EOF) Analysis}

In this subsection, we present additional results of the EOF analysis applied to the highlatitude ionospheric electrostatic potentials in the Northern Hemisphere, generated from the LFM-MIX model at the Quad resolution. Figure 1 displays the scree plot, the mean and the first four EOFs. The scree plot indicates that the first four EOFs can explain approximately $95 \%$ of the total variability in the data. Compared with the curve of the proportion of variance explained for the Dynamics Explorer-2 (DE-2) satellite data and the Super Dual Auroral Radar Network (SuperDARN) data, analyzed in Matsuo et al. (2002); Cousins et al. (2013), respectively, the curve of the proportion of variance explained for the LFM-MIX model output increases more rapidly, with fewer EOFs explaining more variability. This suggests that the real observed data contain more small-scale details than the simulation model output.

The mean and the first four EOFs show similar patterns as those derived from the real observed data (Matsuo et al., 2002; Cousins et al., 2013). These patterns are physically meaningful. The mean electrostatic potential exhibits a typical two-cell ionospheric convection pattern, where the two convection cells are symmetric in shape. The first and second EOFs also have a two-cell structure, which however is asymmetrical, with the latter being more evident. They have the effects of strengthening and weakening the mean convec- 
tion pattern, and/or shaping the symmetric two-cell convection pattern into asymmetrical round and crescent-shaped cells. The third EOF shows two anticorrelated cells between $60^{\circ}$ and $75^{\circ}$ latitude, which indicates expanding of the mean convection pattern toward midlatitudes. The fourth EOF is dominated by a single cell around magnetic midnight and $75^{\circ}$ latitude, playing the role of rotating the mean convection pattern.

\section{S.2.2 Natural Cubic B-spline Basis Functions}
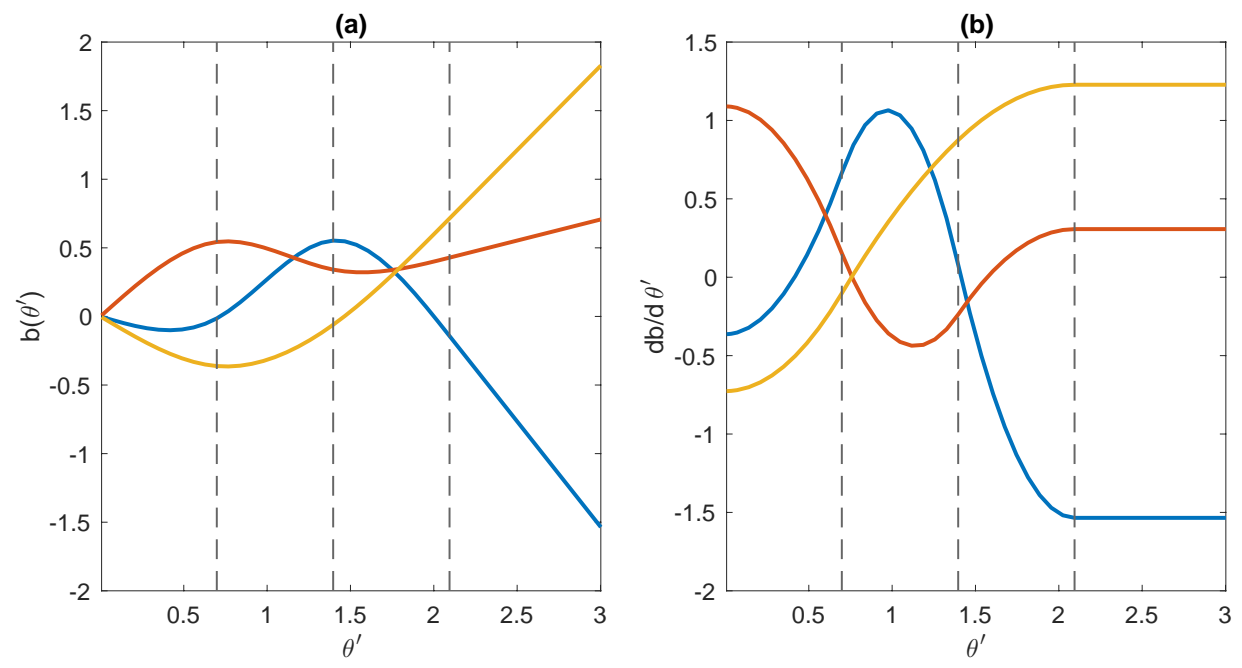

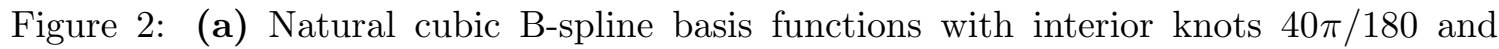
$80 \pi / 180$, and a boundary knot $120 \pi / 180$, indicated by vertical dashed lines; (b) Firstorder derivatives of the natural cubic B-spline basis functions.

Figure 2 displays the natural cubic B-spline basis functions $\left\{b_{i}\left(\theta^{\prime}\right), 0 \leq \theta^{\prime} \leq \pi, i=\right.$ $1, \cdots, r=3\}$ and their first-order derivatives $d b_{i} / d \theta^{\prime}$. The boundary knot is specified as $120 \pi / 180$, where the basis functions are forced to be linear to its right. The enforced linearity makes the model more robust to outliers in lower latitudes, and thus avoids unphysical fitted results. Two interior knots are then chosen uniformly over the range from 0 to $120 \pi / 180$, i.e., at $40 \pi / 180$ and $80 \pi / 180$. If the data are un-stretched from the entire sphere to the high-latitude region of the Northern Hemisphere, the locations of all the knots are effectively $10^{\circ}, 20^{\circ}$ and $30^{\circ}$ co-latitude.

\section{S.2.3 MCMC Diagnostics}

This subsection reports MCMC diagnostics for the application to the high-latitude ionospheric electrostatic potentials. Figure 3 displays the MCMC chains of the parameters 
$\left(\boldsymbol{\eta}_{-0}, \sigma_{2}^{2}, \sigma_{3}^{2}, \sigma_{4}^{2}, 1 / \tau^{2}\right)$ after thinning, and Figures 4 and 5 show the sample autocorrelation functions and the scatter plots of the MCMC samples (not including the burn-in period and after thinning), respectively. Since we have provided a good initial value for the algorithm, all the chains seem to converge successfully. The chains of $\left(\sigma_{2}^{2}, \sigma_{3}^{2}, \sigma_{4}^{2}, 1 / \tau^{2}\right)$ have good mixing rates with low autocorrelation, while those of $\boldsymbol{\eta}_{-0}$ have relatively higher autocorrelation. The high correlation among the MCMC samples of pairs of $\eta_{i}$ 's, indicated in the scatter plots, partially explain the difficulty of sampling $\boldsymbol{\eta}_{-0}$.
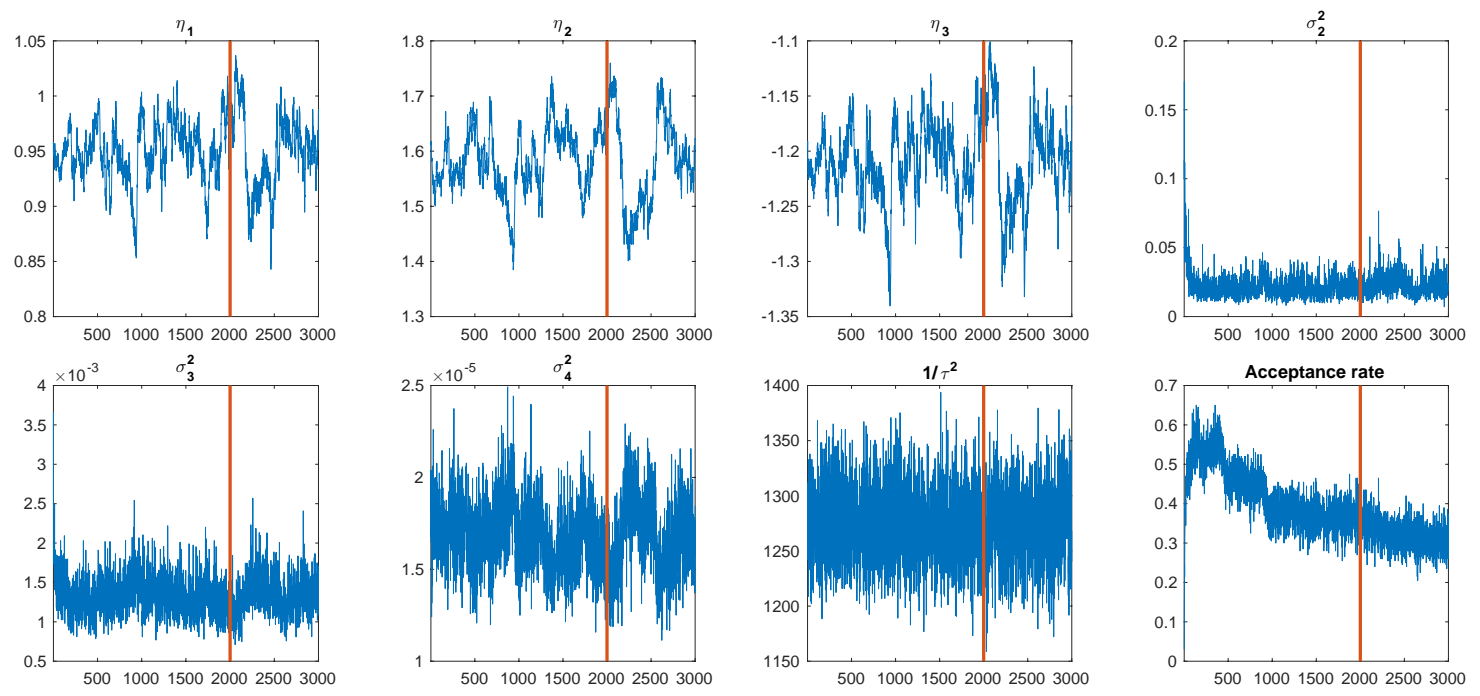

Figure 3: MCMC chains of $\left(\boldsymbol{\eta}_{-0}, \sigma_{2}^{2}, \sigma_{3}^{2}, \sigma_{4}^{2}, 1 / \tau^{2}\right)$ after thinning, and acceptance rate of every 200 adaptive Metropolis steps. Samples to the left of the vertical line are discarded as a burn-in period.

\section{S.2.4 Additional Simulations of Fitted AXING-need Model}

In this subsection, we present additional simulations of the fitted AXING-need model. Figure 6 displays the decomposition of the simulations of the fitted AXING-need model into frequency levels $j=2,3,4$. Each simulation in the first row equals the sum of the simulations from the second to the fourth row in the same column. We can see that the fitted AXING-need model provides a multi-resolution representation, in which higher frequency components capture finer details. Figure 7 shows the sum of the simulation of the fitted AXING-need model and the subtracted large-scale component. We can see that the high-latitude ionospheric convection pattern, mainly contributed by the largescale component, exhibits a typical two-cell morphology and remains dominant in all the nine simulations. There are some differences in fine details among the simulations due to the different simulations of the fitted AXING-need model, which serves as the small-scale 

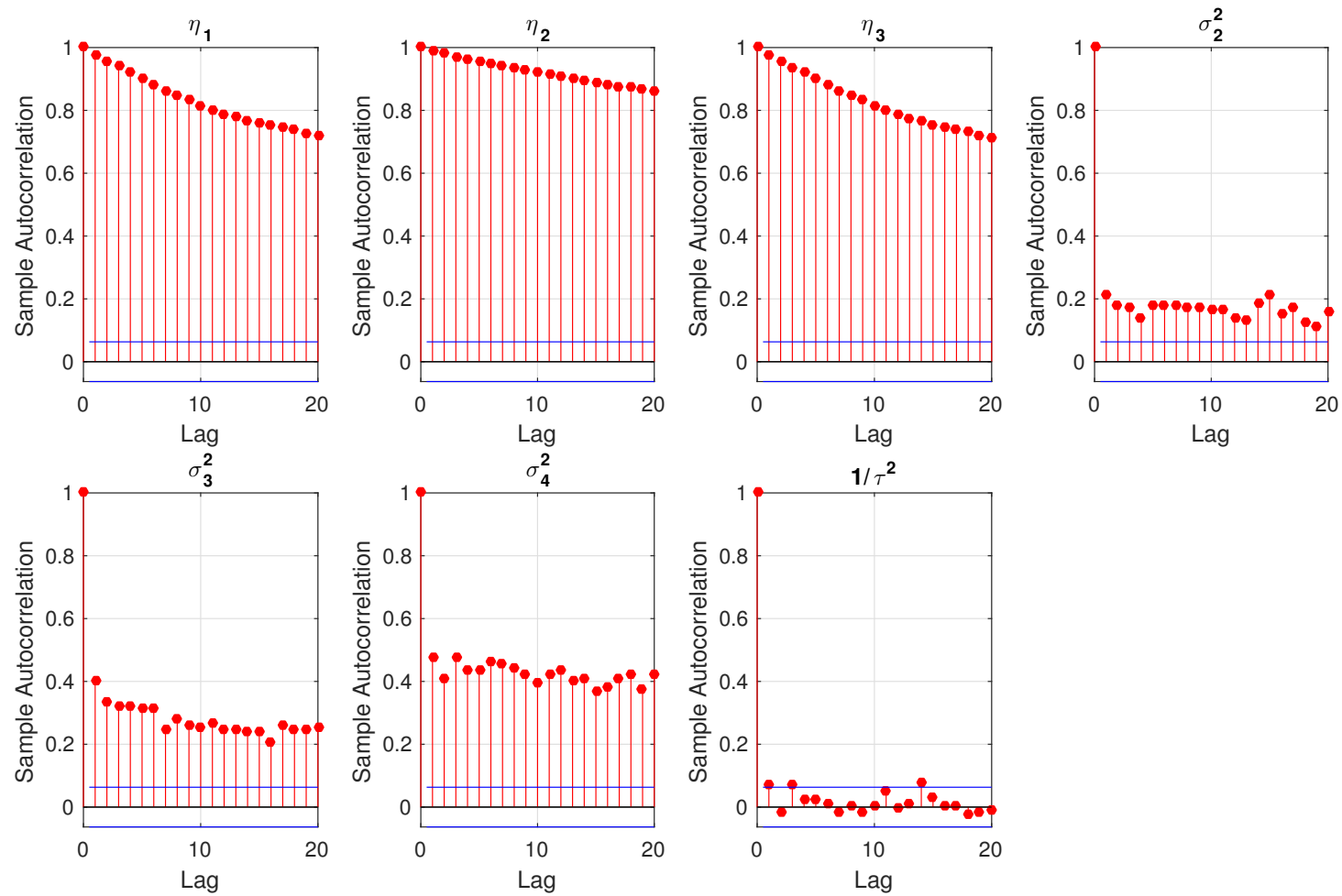

Figure 4: Sample autocorrelation functions of the MCMC samples of $\left(\boldsymbol{\eta}_{-0}, \sigma_{2}^{2}, \sigma_{3}^{2}, \sigma_{4}^{2}, 1 / \tau^{2}\right)$ (not including the burn-in period and after thinning). 


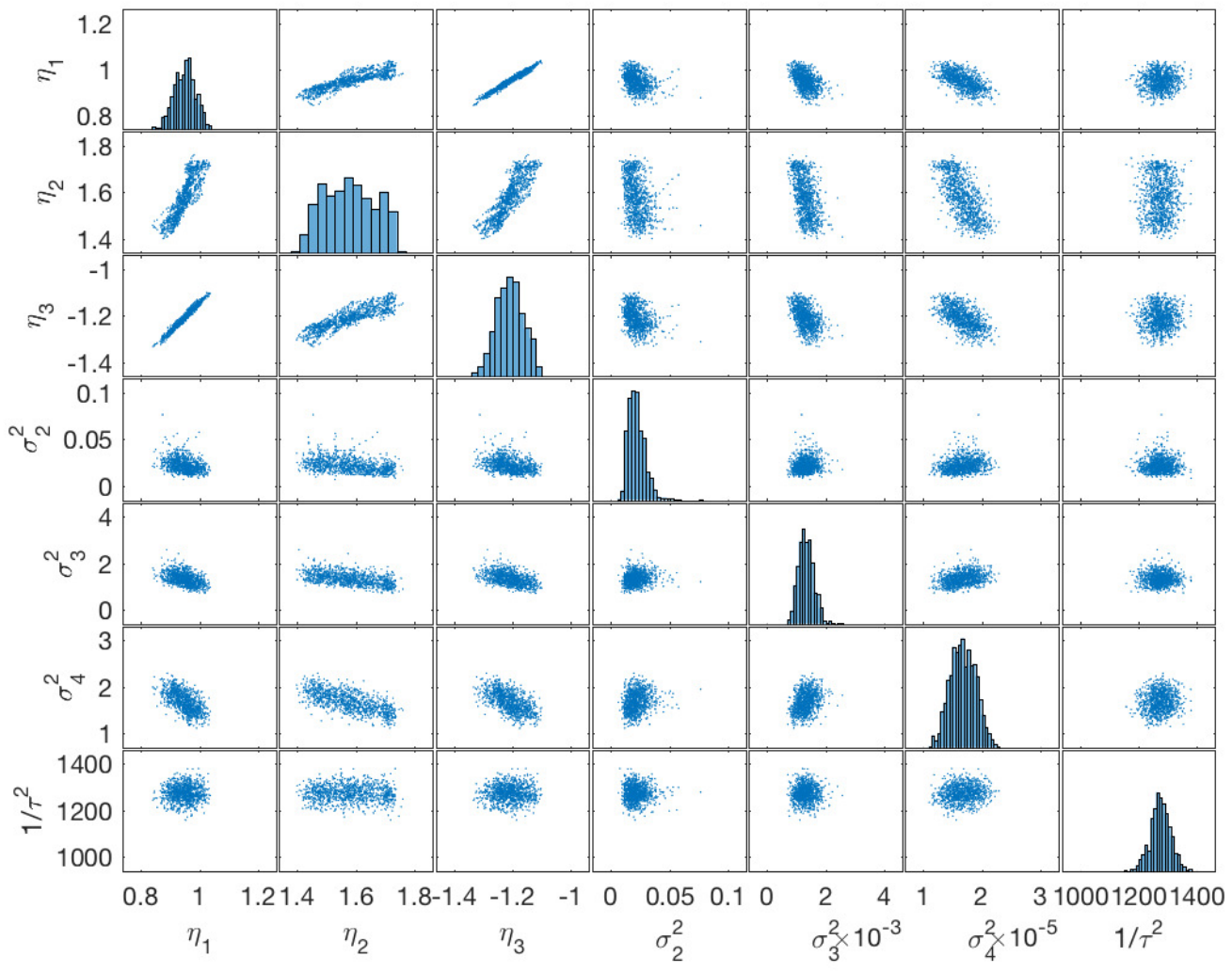

Figure 5: Scatter plots of the MCMC samples of $\left(\boldsymbol{\eta}_{-0}, \sigma_{2}^{2}, \sigma_{3}^{2}, \sigma_{4}^{2}, 1 / \tau^{2}\right)$ (not including the burn-in period and after thinning). 

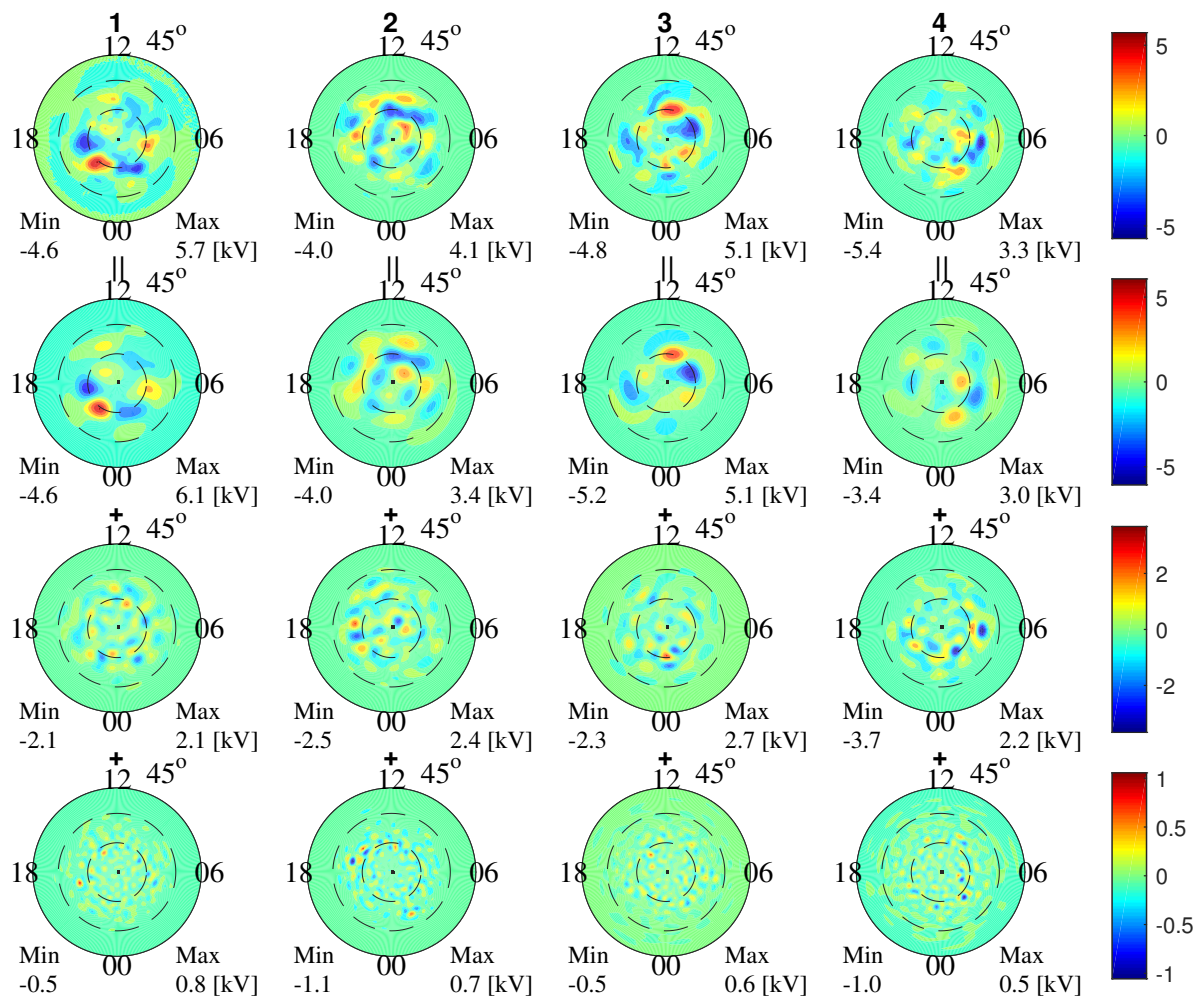

Figure 6: Decomposition of the simulations of the fitted AXING-need model into frequency levels $j=2,3,4$, shown in geomagnetic coordinates. Each simulation in the first row equals the sum of the simulations from the second to the fourth row in the same column, corresponding to $j=2,3,4$, respectively. 

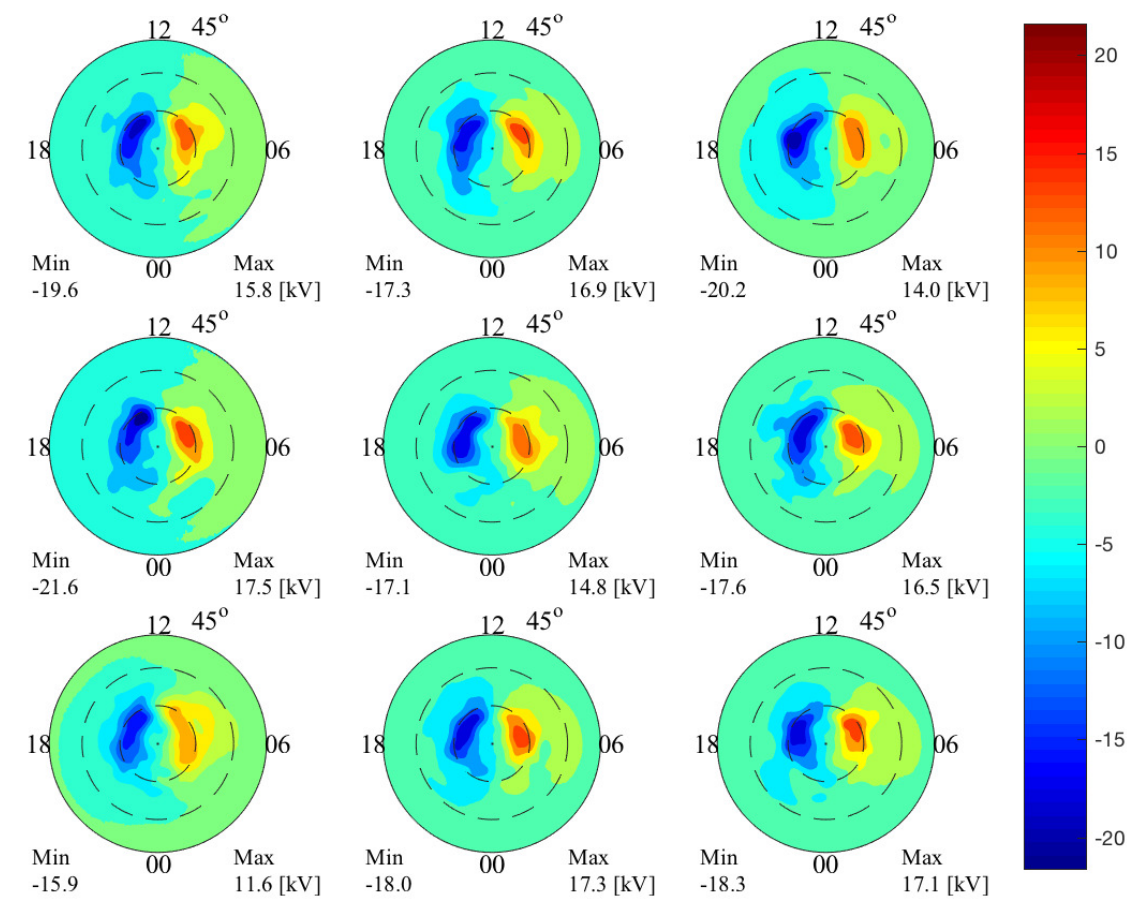

Figure 7: Sum of the simulation of the fitted AXING-need model and the subtracted large-scale component, shown in geomagnetic coordinates. 
component.

\section{S.2.5 Comparable Simulations of Three Candidate Models}

This subsection describes the method of generating comparable simulations of the AXINGneed, Gau-need, and Gau-Matérn models. Recall that the former two share the same form, $\mathbf{Z}=\mathbf{G A} \mathbf{c}+\mathbf{e}$, where each element of $\mathbf{c}$, i.e., $c_{j k}$, follows either a scaled $t$-distribution or a normal distribution. A random sample from a $t$-distribution with $\nu$ degrees of freedom can be generated using the fact that a $t$-distributed random variable has the representation

$$
\frac{Z_{\text {norm }}}{\sqrt{V_{\text {chi }} / \nu}}
$$

where $Z_{\text {norm }} \sim \mathcal{N}(0,1), V_{\text {chi }} \sim \chi^{2}(\nu)$, and they are independent of each other. Thus, the coefficients $c_{j k}$ 's of both models can be generated using the same set of random samples from the standard normal distribution. On the other hand, GRFs can be simulated based on the Cholesky decomposition. Suppose that the covariance matrix of a GRF evaluated at $n$ sampling locations can be decomposed as $\boldsymbol{\Sigma}=\mathbf{L} \mathbf{L}^{\mathrm{T}}$, where $\mathbf{L}$ is a lower triangular matrix with positive diagonal elements. Then, the vector $\mathbf{L u}$ is a simulation of the GRF at the sampling locations, where the vector of random deviates $\mathbf{u}$ is generated from $\mathcal{N}\left(\mathbf{0}, \mathbf{I}_{n}\right)$. Thus, given a simulation of the Gau-need model, denoted by $\mathbf{Z}$, we can recover the latent random deviates by $\mathbf{L}^{-1} \mathbf{Z}$. They are used to generate comparable simulations of the Gau-Matérn model based on the Cholesky decomposition of the corresponding covariance matrix.

\section{S.2.6 Cross-validation Training and Test Set Separation}

In the cross-validation procedure, each time the data set is separated into training and test sets, where the former is specified according to the spatial coverage of the SuperDARN data, represented in geomagnetic coordinates. In the high-latitude region of the Northern Hemisphere, the observations tend to be restricted to higher latitudes (latitude $\geq 65^{\circ}$ ) (see Figure 1 in Cousins et al. (2013)), and data gaps typically occur within a block of magnetic local time (MLT). The left panel of Figure 8 displays an example of the spatial coverage of the SuperDARN data in the high-latitude region of the Northern Hemisphere at a particular time (http://vt.superdarn.org/tiki-index.php), which exhibits the aforementioned characteristics. Thus, we randomly select 1000 locations as the observed ones for model fitting, with latitudes higher than $65^{\circ}$ and outside a randomly selected $6 \mathrm{~h}$ block of MLT (i.e., $90^{\circ}$ in geomagnetic longitude), and hold out the remaining locations to test the predictive performance. The right panel of Figure 8 shows the observed locations for one of the cross-validation replications. 

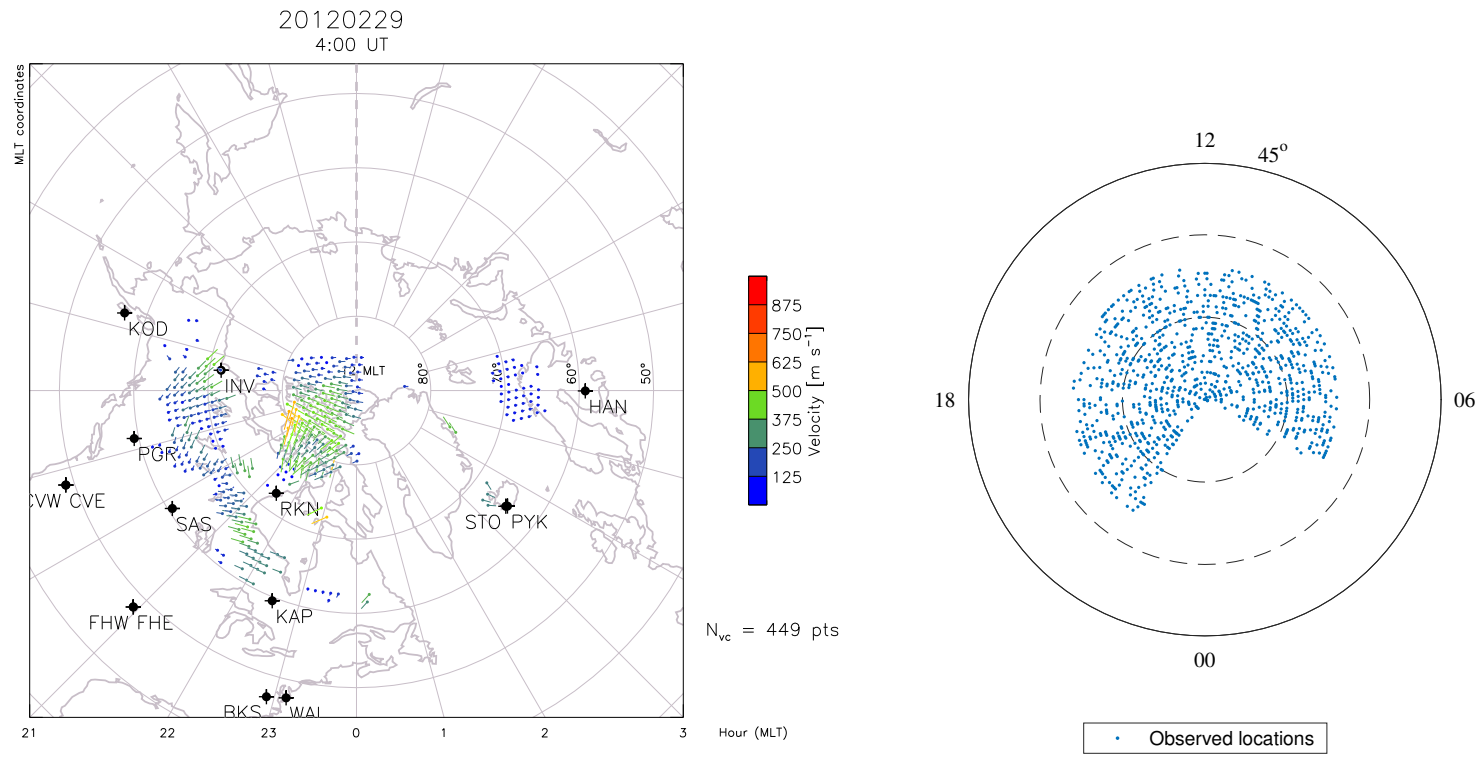

Figure 8: Left: Spatial coverage of the SuperDARN data in the high-latitude region of the Northern Hemisphere at 4:00 UT on Feb 29th, 2012; Right: Observed locations for one of the cross-validation replications.

\section{S.3 Additional Figures}

This section provides additional figures mentioned in the main text.

\section{References}

Baldi, P., Kerkyacharian, G., Marinucci, D. and Picard, D. (2009) Asymptotics for spherical needlets. The Annals of Statistics, 37, 1150-1171.

Cousins, E. D. P., Matsuo, T. and Richmond, A. D. (2013) Mesoscale and large-scale variability in high-latitude ionospheric convection: Dominant modes and spatial/temporal coherence. Journal of Geophysical Research: Space Physics, 118, 7895-7904.

Gneiting, T. and Raftery, A. E. (2007) Strictly proper scoring rules, prediction, and estimation. Journal of the American Statistical Association, 102, 359-378.

Grimit, E. P., Gneiting, T., Berrocal, V. J. and Johnson, N. A. (2006) The continuous ranked probability score for circular variables and its application to mesoscale forecast ensemble verification. Quarterly Journal of the Royal Meteorological Society, 132, 29252942. 

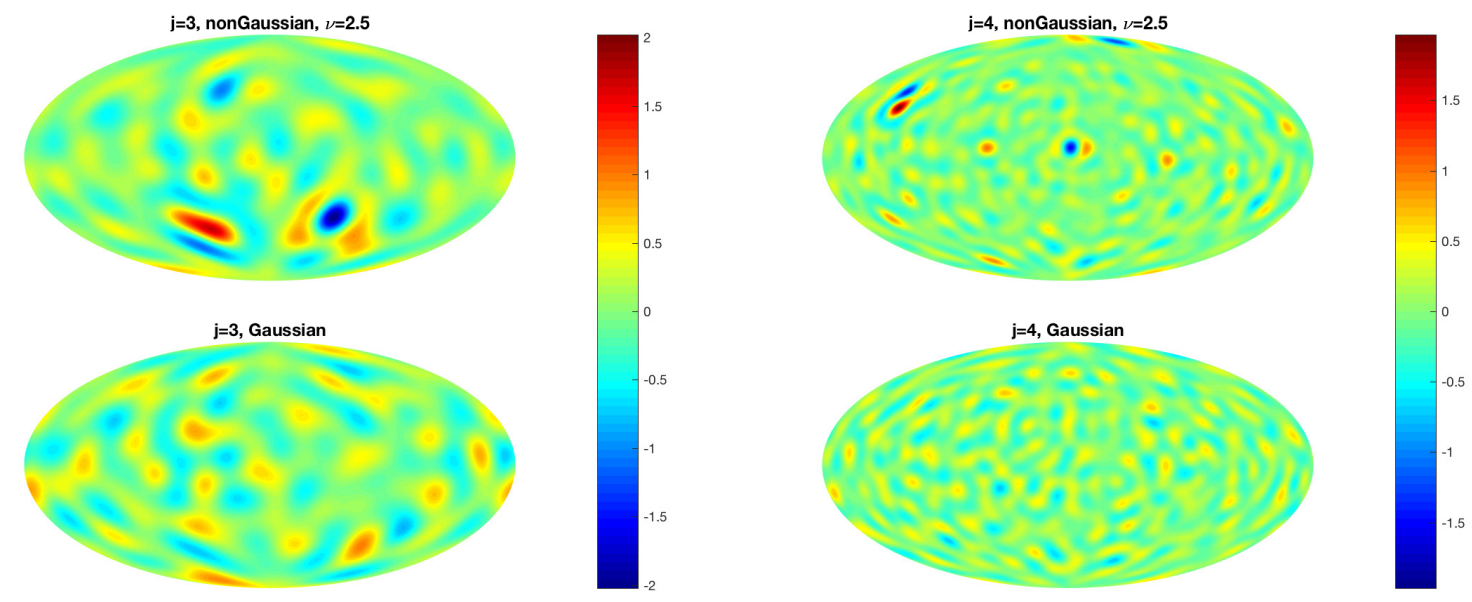

Figure 9: Comparison of simulations of $X$ with $t$-distributed and normally distributed $c_{j k}$ 's, projected onto frequency levels $j=3$ (left panel) and $j=4$ (right panel).
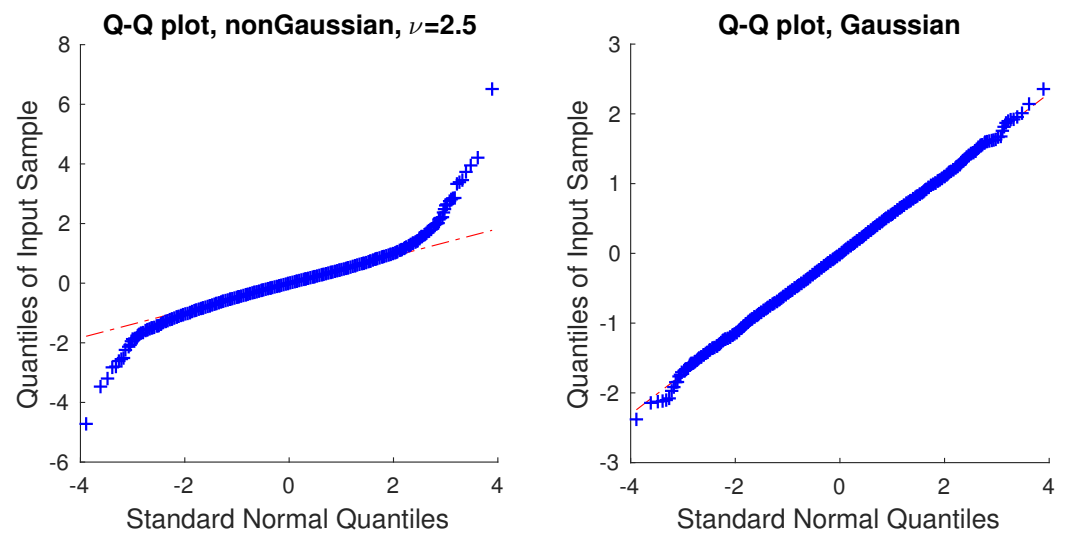

Figure 10: Comparison of Q-Q plots of 10,000 i.i.d. simulations of $X$ with $t$-distributed and normally distributed $c_{j k}$ 's at a specific location. 
Krüger, F., Lerch, S., Thorarinsdottir, T. L. and Gneiting, T. (2016) Probabilistic forecasting and comparative model assessment based on Markov Chain Monte Carlo output. arXiv preprint arXiv:1608.06802.

Marinucci, D. and Peccati, G. (2011) Random fields on the sphere: Representation, limit theorems and cosmological applications, vol. 389 of London Mathematical Society Lecture Note Series. Cambridge: Cambridge University Press.

Matsuo, T., Richmond, A. D. and Nychka, D. W. (2002) Modes of high-latitude electric field variability derived from DE-2 measurements: Empirical Orthogonal Function (EOF) analysis. Geophysical Research Letters, 29, 11-1-11-4.

Womersley, R. S. (2015) Efficient spherical designs with good geometric properties. http: //web.maths.unsw.edu.au/ rsw/Sphere/EffSphDes/. 